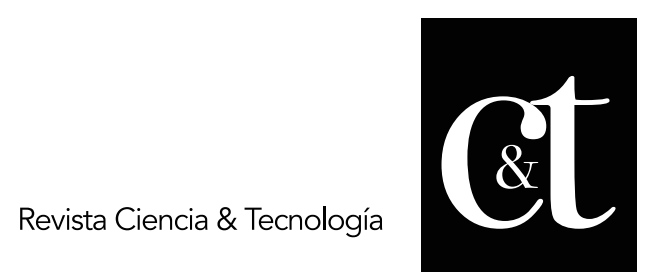

No. 32, 31 de octubre de 2021

ISSN impreso: 1390 - 6321

ISSN online: 2661 - 6734

\title{
Liderazgo empresarial en tiempos COVID-19: Caso de estudio Cooperativa de Ahorro y Crédito El Sagrario Ltda.
}

\section{Business leadership in COVID-19 times: Case study Cooperativa de Ahorro y Crédito El Sagrario Ltda.}

\author{
Rommel Velastegui ${ }^{1}$ \\ rs.velastegui@uta.edu.ec \\ https://orcid.org/0000-0001-6653-7933 \\ Emilio Freire ${ }^{2}$ \\ efreire3912@uta.edu.ec \\ https://orcid.org/0000-0003-3607-0863 \\ María José Mayorga ${ }^{3}$ \\ mariajmayorga@uta.edu.ec \\ https://orcid.org/0000-0003-1897-739X \\ Luis Tello 4 \\ Itello@uta.edu.ec \\ https://orcid.org/0000-0003-1924-303X
}

Recibido: 28/6/2021, Aceptado: 28/9/2021

\begin{abstract}
RESUMEN
El presente estudio de caso tiene como finalidad la aplicación del Test de Liderazgo Situacional de Hersey y Blanchard a 52 colaboradores de una institución financiera, el documento cuenta con 12 dimensiones de estudio para lograr definir el tipo de liderazgo empresarial y como este puede afectar en los procesos corporativos debido a los cambios externos abruptos tal es el caso del COVID - 19; el resultado de la aplicación del test arroja resultados en Q2 que falta dirección y apoyo; sin embargo en la institución se toma la teoría del liderazgo de Goleman proponiendo un liderazgo empresarial de Couch y Afiliativo con esta propuesta se ha obtenido mantener al $100 \%$ de los colaboradores en las actividades laborales reestructurando actividades, garantizando incremento de un $25 \%$ en la calidad de vida, afectando $3 \%$ los fatores de riesgo psicosocial a los que están expuestos y logrando que se incremente en $16 \%$ el desempeño laboral convirtiendo a la empresa en una institución resiliente.
\end{abstract}

\footnotetext{
${ }^{1}$ Magíster en Gestión de operaciones, Universidad Técnica de Ambato, Ecuador

${ }^{2}$ Psicólogo industrial, Universidad Técnica de Ambato, Ecuador

${ }^{3}$ Magíster en Contabilidad y Auditoría, Universidad Técnica de Ambato, Ecuador

${ }^{4}$ Magíster en Gestión del Talento Humano, Universidad Técnica de Ambato, Ecuador
} 


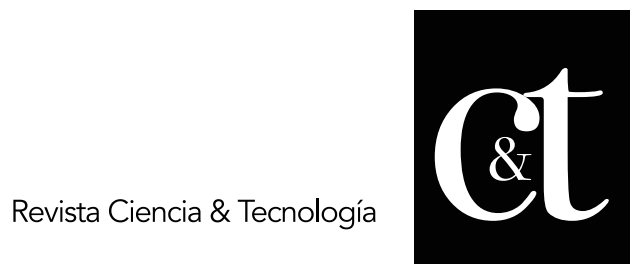

No. 32, 31 de octubre de 2021

ISSN impreso: 1390 - 6321

ISSN online: 2661 - 6734

\title{
Palabras clave
}

Liderazgo empresarial, COVID- 19, Hersey y Blanchard, Daniel Goleman

\begin{abstract}
The purpose of this case study is to apply the Hersey and Blanchard Situational Leadership Test to 52 employees of a financial institution, the document has 12 study dimensions to define the type of business leadership and how this can affect the corporate processes due to abrupt external changes such is the case of COVID - 19; The result of the application of the test shows results in Q2 that lack direction and support; However, in the institution, the theory of Goleman's leadership is taken, proposing a business leadership of Couch and Affiliative with this proposal, it has been obtained to maintain $100 \%$ of the collaborators in work activities by restructuring activities, guaranteeing an increase of $25 \%$ in the quality of life, affecting $3 \%$ of the psychosocial risk factors to which they are exposed and achieving a $16 \%$ increase in work performance, turning the company into a resilient institution.
\end{abstract}

\section{Keywords}

Business Leadership, COVID-19, Hersey and Blanchard, Daniel Goleman

\section{Introducción}

En la actualidad debido a los cambios alíferos de las organizaciones el liderazgo empresarial es muy importante para ser competitivo en un mercado muy competitivo, por medio de los líderes las empresas logran ser trascendentales, consiguen desarrollar colaboradores competentes, optimizan procesos productivos y de servicio permitiendo superar los objetivos propuestos por las instituciones (García, 2015).

Debido a esta nueva normalidad que vivimos ocasionada por el virus las acciones operativas en las organizaciones se deben desarrollar a través de acciones innovadoras y dinámicas, con el fin de mantener a las empresas resilientes a cambios repentinos (Turbay, 2013).

Liderazgo empresarial no solo abarcar a la alta gerencia sino todo lo que conlleva clima y cultura organizacional, calidad de vida, necesidades de colaboradores, empoderamiento de los integrantes de la empresa para que se fomente el proceso de liderazgo empresarial (Vu, 2020).

El liderazgo empresarial a nivel mundial en tiempos de COVID-19 es fundamental en para el desarrollo de la gestión organizacional, sobre todo considerando el desarrollo potencial de la organización, es allí donde el líder cumple un rol importante para el surgimiento o declive de la institución (Izquierdo, Novillo, \& Mocha, 2017). Las empresas que más han sobresalido internacionalmente, Facebook, Google, y a nivel nacional Plasticaucho, Cervecería Nacional entre otras logran incentivar la creatividad de los colaboradores, fomentando una excelente calidad de vida organizacional, motivando a los colaboradores el sentimiento de orgullo para aportar conocimientos en esas instituciones (Rexhepi, Sogojeva, Rexhepi, \& Lekiqi, 2020). 


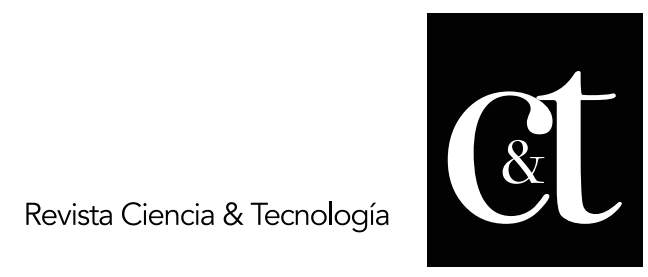

No. 32, 31 de octubre de 2021

ISSN impreso: 1390 - 6321

ISSN online: 2661 - 6734

La responsabilidad social empresarial (RSE) por tiempos de COVID-19 es muy importante para el cuidado de los colaboradores que al reestructurar procesos son los nuevos líderes, además en la actualidad las empresas tienen la necesidad de preparar a nuevos líderes quienes serán los encargados del autocuidado de la salud y de la detección de inconformidades en los puesto de trabajo (Ward, 2012).

Es por eso que el siguiente caso de estudio tiene como propósito valorar el liderazgo empresarial en tiempos de COVID-19 y su influencia en los colaboradores de la Cooperativa de Ahorro y Crédito El Sagrario Ltda. de la ciudad de Ambato, las fuerzas externas en el Desarrollo Organizacional hace que reaccione con presura las empresas y el liderazgo empresarial es indispensable para el desarrollo de las organizaciones teniendo en cuenta las habilidades gerenciales, el autoestima y la seguridad en su desempeño de cada uno de los colaboradores (Mantulak \& Hernández, 2018).

La investigación propone un análisis y mejoramiento del liderazgo empresarial tomando como referencia la situación actual en la que se encuentran los colaboradores con la institución sumándole un factor externo inesperado COVID19 a la que el estilo de liderazgo organizacional debe reaccionar inmediatamente sino quiere colapsar (Rexhepi, Sogojeva, Rexhepi, \& Lekiqi, 2020).

Los resultados obtenidos sobre el estilo de liderazgo han logrado desarrollar medidas preventivas para los colaboradores que tienen referencia con riesgos psicosociales, estrés laboral y otras que permitan el mejor desarrollo personal y profesional de los colaboradores sin interrupción dentro organización. (Goleman, 2016)

\section{Método y descripción del caso}

El siguiente análisis de caso se ha desarrollado bajo la siguiente metodología propuesta por el grupo de investigación:

\subsection{Población de estudio}

Para llevar a cabo el proceso de investigación se ha tomado una población de estudio el cual abarca la totalidad de los colaboradores de la Cooperativa de Ahorro y Crédito El Sagrario Ltda., siendo 52 personas, se ha tomado un único criterio de inclusión a la totalidad de los colaboradores de la oficina matriz de la empresa; debido que la población en estudio es pequeña, teniendo 27 colaboradores de género masculino y 25 de género femenino, con un rango de edad que se encuentra entre los 23 a los 59 años de edad (Meliá, y otros, 2016).

\subsection{Análisis y recolección de información}

En el proceso investigativo se utilizó la herramienta llamada test de Liderazgo Situacional de Hersey y Blanchard para la evaluación de liderazgo empresarial, (Hersey \& Blanchard, 2005).

El documento contiene 12 dimensiones indicadas en la Tabla 1, donde los colaboradores a ser evaluados tienen 4 opciones dentro cada dimensión como se indica en la Tabla 3. 


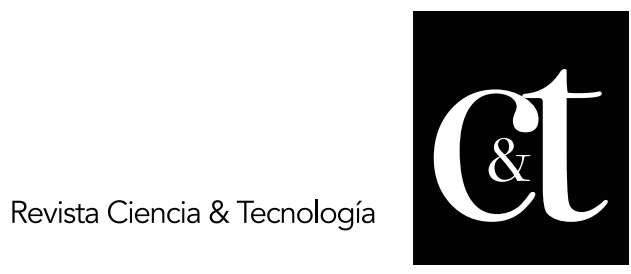

No. 32, 31 de octubre de 2021

ISSN impreso: 1390 - 6321

ISSN online: 2661 - 6734

Las opciones de elección son simbolizada por una escala valorativa de Likert, el tiempo de aplicación que considera Hersey y Blanchard se aproxima a 30 minutos por la complejidad de las dimensiones. (Pinto \& Gutiérrez, 2015).

Tabla 1. Dimensiones test de liderazgo Hersey y Blanchard

\begin{tabular}{|c|c|}
\hline N. & Dimensiones \\
\hline 1 & $\begin{array}{l}\text { Sus colaboradores no están respondiendo en el último tiempo a } \\
\text { su conversación amistosa y a su obvia preocupación por su } \\
\text { bienestar. Su desempeño está declinando rápidamente. }\end{array}$ \\
\hline 2 & $\begin{array}{l}\text { El desempeño observable de su grupo está mejorando. Usted se } \\
\text { ha estado asegurando que todos los miembros estén conscientes } \\
\text { de sus responsabilidades y los estándares esperados de } \\
\text { desempeño. }\end{array}$ \\
\hline 3 & $\begin{array}{l}\text { Los miembros de su grupo son incapaces de resolver un problema } \\
\text { por sí mismos. Usted los ha dejado normalmente solos. El } \\
\text { desempeño y las relaciones interpersonales del grupo han sido } \\
\text { buenas. }\end{array}$ \\
\hline 4 & $\begin{array}{l}\text { Usted está considerando un cambio. Sus colaboradores tienen un } \\
\text { excelente historial de logros. Ellos reconocen la necesidad de } \\
\text { cambios. }\end{array}$ \\
\hline 5 & $\begin{array}{l}\text { El desempeño de su grupo ha estado decayendo durante los } \\
\text { meses recientes. Los integrantes no están preocupados con el } \\
\text { logro de los objetivos. La redefinición de roles y responsabilidades } \\
\text { ha sido de utilidad en el pasado. Ha sido necesario estarles } \\
\text { recordando constantemente que realicen sus tareas a tiempo. }\end{array}$ \\
\hline 6 & $\begin{array}{l}\text { Usted ha ingresado a una organización manejada en forma } \\
\text { eficiente. El anterior administrador controlaba estrechamente la } \\
\text { situación. Usted quiere mantener una situación productiva, sin } \\
\text { embargo, le gustaría comenzar a humanizar el entorno. }\end{array}$ \\
\hline 7 & $\begin{array}{l}\text { Usted está considerando el cambiar a una estructura que será } \\
\text { nueva para su grupo. Los miembros del grupo han hecho } \\
\text { sugerencias acerca de los cambios requeridos. El grupo ha sido } \\
\text { productivo y ha demostrado flexibilidad en sus operaciones. }\end{array}$ \\
\hline 8 & $\begin{array}{l}\text { El desempeño del grupo y las relaciones interpersonales son } \\
\text { buenas, usted se siente un tanto inseguro acerca de su falta de } \\
\text { dirección del grupo. }\end{array}$ \\
\hline 9 & $\begin{array}{l}\text { Su jefe lo ha designado para encabezar una fuerza de tarea (task } \\
\text { force) que está sumamente retrasada en hacer las }\end{array}$ \\
\hline & $\begin{array}{l}\text { recomendaciones requeridas para un cambio. El grupo no está } \\
\text { claro con respecto a sus metas. La asistencia a las reuniones ha } \\
\text { sido baja. Sus reuniones se han transformado más bien en } \\
\text { encuentros sociales. Potencialmente ellos tienen el talento } \\
\text { necesario para ayudar. }\end{array}$ \\
\hline 10 & $\begin{array}{l}\text { Sus colaboradores, que normalmente son capaces de asumir } \\
\text { responsabilidades, no están respondiendo a su reciente definición } \\
\text { de estándares. }\end{array}$ \\
\hline 11 & $\begin{array}{l}\text { Usted ha sido promovido a una nueva posición. El supervisor } \\
\text { anterior no estaba involucrado en los asuntos del grupo. El grupo } \\
\text { ha manejado adecuadamente sus tareas y dirección. Las } \\
\text { interrelaciones del grupo son buenas. }\end{array}$ \\
\hline
\end{tabular}




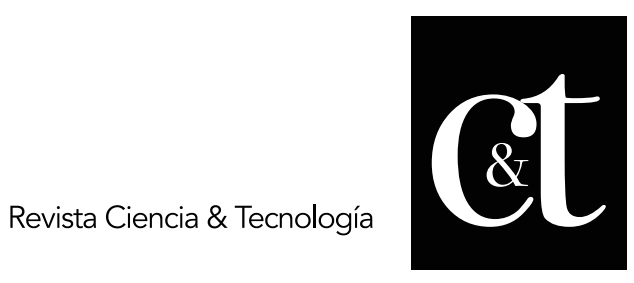

No. 32, 31 de octubre de 2021

ISSN impreso: 1390 - 6321

ISSN online: 2661 - 6734

Informaciones recientes indican que existen algunas dificultades internas entre los colaboradores. El grupo tiene un registro notable de logros. Los miembros han mantenido efectivamente metas de largo plazo. Han trabajado en armonía durante el último año. Todos están bien calificados para las tareas.

Fuente: (Hersey \& Blanchard, 2005)

\subsection{Metodología de evaluación de la prueba de Liderazgo}

Una vez que se identificó la población de estudio para aplicar el test se consideró los siguientes aspectos: a) elegir un espacio donde no existan distractores con la finalidad que la información recolectada sea la más cercana a la realidad; b) dividir en grupos según el área o departamento de trabajo al que pertenezca; c) considerar una sola aplicación del test a cada uno de los colaboradores; d) socializar las instrucciones de la prueba de liderazgo; e) el evaluador sería una guía y apoyo en caso de que algún colaborador tenga dudas sobre alguna dimensión o terminología del test. (Thompson \& Glaso, 2018).

El evaluador juega un papel fundamental para que no exista una tendencia ni positiva ni negativa de la información o los resultados que se pretende obtener, la prueba ha permitido al colaborador tener un conocimiento leve de la función del líder de la organización (Ali Raza \& Sikandar, 2018).

\subsection{Validación de datos a través de Software SPSS}

Una vez que se ha finalizado el proceso de evaluación de la prueba de liderazgo tenemos: a) recolección de la información; b) validación de los resultados obtenidos, que esta información no tenga tendencia; c) exclusión de información con cierto tipo de tendencia o errores que el investigador considere; d) consolidación de datos a través del software estadístico SPSS; e) validación de la información (Ariawan \& Wahyuni, 2019).

El proceso de validación se da para poder conocer que los datos recopilados son ordinales, y para poder realizar dentro de la investigación pruebas estadísticas en caso de requerirlo. (IBM Corporation, 2012).

2.5 Elección del cuadrante Q para definir el estilo de liderazgo.

Los resultados de la suma de las Dimensiones, según las opciones de respuesta de los colaboradores se ven reflejados en la Tabla 
Tabla 2 Cuadrantes Q de resultados de la prueba de Liderazgo Situacional de Hersey y Blanchard

\begin{tabular}{|c|c|}
\hline $\begin{array}{l}\text { C } \\
\text { ua } \\
\text { dr } \\
\text { an } \\
\text { te }\end{array}$ & Comportamiento \\
\hline Q1 & $\begin{array}{l}\text { Alto en tarea y bajo en } \\
\text { relacionamiento }\end{array}$ \\
\hline Q2 & $\begin{array}{l}\text { Alto en tarea y alto en } \\
\text { relacionamiento }\end{array}$ \\
\hline Q3 & $\begin{array}{c}\text { Alto en relacionamiento y bajo en } \\
\text { tarea }\end{array}$ \\
\hline Q4 & $\begin{array}{c}\text { Bajo en relacionamiento y bajo en } \\
\text { tarea }\end{array}$ \\
\hline
\end{tabular}

Fuente: (Hersey \& Blanchard, 2005)

\section{Resultados y discusión del caso}

La obtención de resultados y discusión del caso de estudio se da a través de los siguientes pasos:

3.1. Datos sociodemográficos de la población

Los datos sociodemográficos de la población son importantes mencionar debido que de acuerdo con la pirámide de necesidades de Maslow el estilo de liderazgo desde una perspectiva de una persona de una edad de 23 años no va a ser la misma que de una persona de 59 años; es por eso que se ha considerado criterios para evaluación de datos sociodemográficos: género y edad los cuales se ven reflejados en la Tabla 3 (Makovsky, 2014).

Tabla 3. Datos sociodemográficos de los colaboradores

\begin{tabular}{ccc}
\hline Género & $\begin{array}{c}\text { Número de } \\
\text { colaboradores }\end{array}$ & Edad \\
\hline Masculino & 27 & 23 a \\
Femenino & 25 & 59 \\
& años
\end{tabular}

Fuente: (Cooperativa de Ahorro y Crédito San Francisco Ltda., 2020)

3.2. Dimensiones de estudio Test de Liderazgo Situacional de Hersey y Blanchard.

Los resultados obtenidos en base a la aplicación de la prueba de liderazgo situacional de Hersey y Blanchard se demuestran de acuerdo con las 12 dimensiones indicadas en la Tabla 1; para explicación del caso se ha tomado en cuenta a la dimensión más relevante siendo la dimensión 5 como se muestra en la Tabla 4. 
Tabla 4. Test Liderazgo. Pregunta No.5.

Dimensión 5. El desempeño de su grupo ha estado decayendo durante los meses recientes. Los integrantes no están preocupados con el logro de los objetivos. La redefinición de roles y responsabilidades ha sido de utilidad en el pasado. Ha sido necesario estarles recordando constantemente que realicen sus tareas a tiempo.

\begin{tabular}{|c|c|c|c|c|}
\hline Opciones & f & $\%$ & $\begin{array}{l}\% \\
\text { v } \\
\text { á } \\
\text { I } \\
\text { i } \\
\text { d } \\
\text { o }\end{array}$ & $\begin{array}{c}\% \\
\text { ac } \\
\text { u } \\
\text { m } \\
\text { ul } \\
\text { a } \\
\text { d } \\
\text { o }\end{array}$ \\
\hline $\begin{array}{l}\text { Incorporar las recomendaciones del } \\
\text { grupo, pero ver que se cumplan los } \\
\text { objetivos. }\end{array}$ & 9 & $\begin{array}{l}1 \\
7 \\
3 \\
3\end{array}$ & $\begin{array}{l}1 \\
7 \\
3\end{array}$ & $\begin{array}{r}17 \\
, 3\end{array}$ \\
\hline $\begin{array}{l}\text { Redefinir los } \\
\text { responsabilidades } \\
\text { cuidadosamente. }\end{array}$ & $\begin{array}{l}4 \\
1\end{array}$ & $\begin{array}{l}7 \\
8 \\
\prime \\
8\end{array}$ & $\begin{array}{l}7 \\
8 \\
\prime \\
8\end{array}$ & $\begin{array}{l}96 \\
2\end{array}$ \\
\hline $\begin{array}{l}\text { Permitir involucramiento del grupo } \\
\text { en la determinación de roles, pero no } \\
\text { siendo demasiado directivo }\end{array}$ & 2 & $\begin{array}{l}3 \\
8\end{array}$ & $\begin{array}{l}3 \\
8\end{array}$ & $\begin{array}{l}10 \\
0, \\
0\end{array}$ \\
\hline Total & $\begin{array}{l}5 \\
2\end{array}$ & $\begin{array}{l}1 \\
0 \\
0 \\
\prime \\
0\end{array}$ & $\begin{array}{l}1 \\
0 \\
0 \\
1 \\
0\end{array}$ & \\
\hline
\end{tabular}

Fuente: (Cooperativa de Ahorro y Crédito San Francisco Ltda., 2020)

n Incorporar las recomendaciones del grupo, pero ver que se cumplan los objetivos.

$\square$ Redefinir los roles y responsabilidades y supervisar cuidadosamente.

- Permitir involucramiento del grupo en la determinación de roles, pero no siendo demasiado directivo

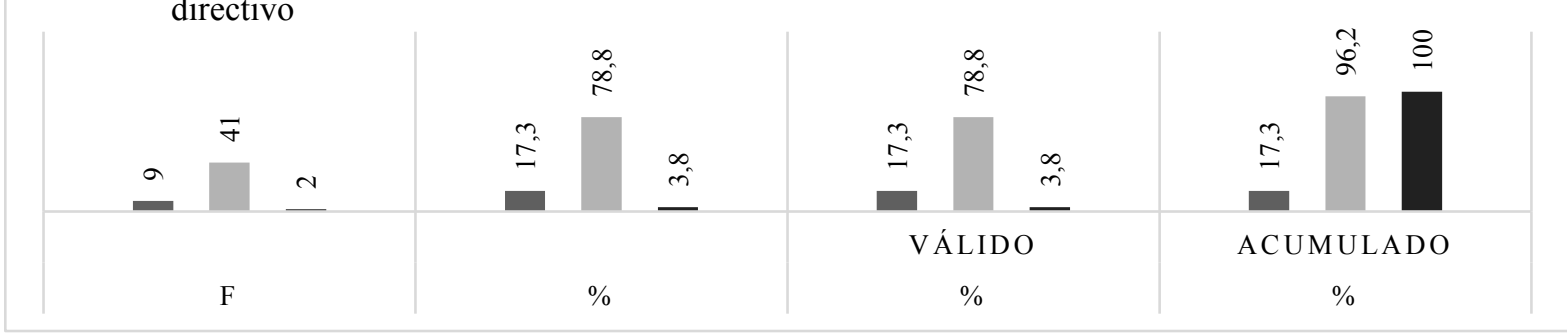

Figura 1 Test Liderazgo. Dimensión N 5.

Fuente: SPSS Caso de estudio:

(Cooperativa de Ahorro y Crédito San Francisco Ltda., 2020) 
La Figura 1 muestra los resultados de las opciones que tuvieron los colaboradores dentro de la Dimensión 5, siendo la más relevante "Redefinir los roles y responsabilidades y supervisar cuidadosamente" con un porcentaje de $78.8 \%$ equivalente a una frecuencia de 41 colaboradores que eligieron esta opción.

\subsection{Resultados globales de la prueba de Liderazgo Situacional de Hersey y Blanchard}

Una vez que se obtuvo los resultados de las opciones de cada una de las Dimensiones, se procede a calcular a través de la metodología (Hersey \& Blanchard, 2005), donde logra se conoce en que cuadrante (Q) se encuentran los colaboradores; el cuadrante $Q$ es el resultado de los tipos de Liderazgo el cual percibe el colaborador; el resultado del estilo de liderazgo es el cual cada colaborador necesita para desarrollarse adecuadamente en su puesto de trabajo (Vu, 2020)-

Tabla 5. Resultados globales de la prueba de Liderazgo Situacional de Hersey y Blanchard

\begin{tabular}{|c|c|c|}
\hline $\begin{array}{l}\text { C } \\
\text { ua } \\
\text { dr } \\
\text { an } \\
\text { te }\end{array}$ & Comportamiento & Frecuencia \\
\hline$\overline{\mathrm{Q} 1}$ & $\begin{array}{l}\text { Alto en tarea y bajo en } \\
\text { relacionamiento }\end{array}$ & 17 \\
\hline Q2 & $\begin{array}{l}\text { Alto en tarea y alto en } \\
\text { relacionamiento }\end{array}$ & 28 \\
\hline Q3 & $\begin{array}{c}\text { Alto en relacionamiento y bajo en } \\
\text { tarea }\end{array}$ & 7 \\
\hline Q4 & $\begin{array}{l}\text { Bajo en relacionamiento y bajo en } \\
\text { tarea }\end{array}$ & 0 \\
\hline
\end{tabular}

Fuente: (Hersey \& Blanchard, 2005)

Los resultados obtenidos son Q1: Alto en tarea y bajo en relación a 17 colaboradores (33\%); Q2: Alto en tarea y alto en relacionamiento correspondiente a 28 colaboradores (54\%); Q3: Alto en relacionamiento y bajo en tarea correspondiente a 7 colaboradores $(13 \%)$ y Q4: Bajo en relacionamiento y bajo en tarea ningún colaborador $(0 \%)$, los porcentajes y frecuencias se reflejan en la Figura 2. (Cooperativa de Ahorro y Crédito San Francisco Ltda., 2020) 


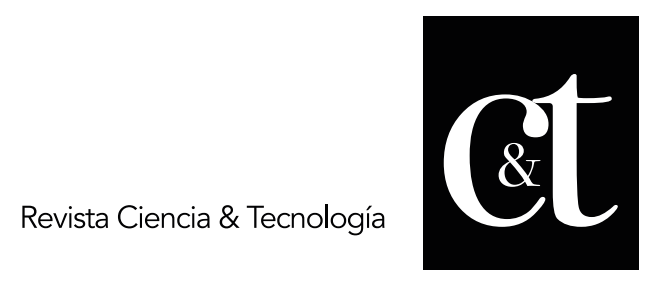

No. 32, 31 de octubre de 2021

ISSN impreso: 1390 - 6321

ISSN online: 2661 - 6734

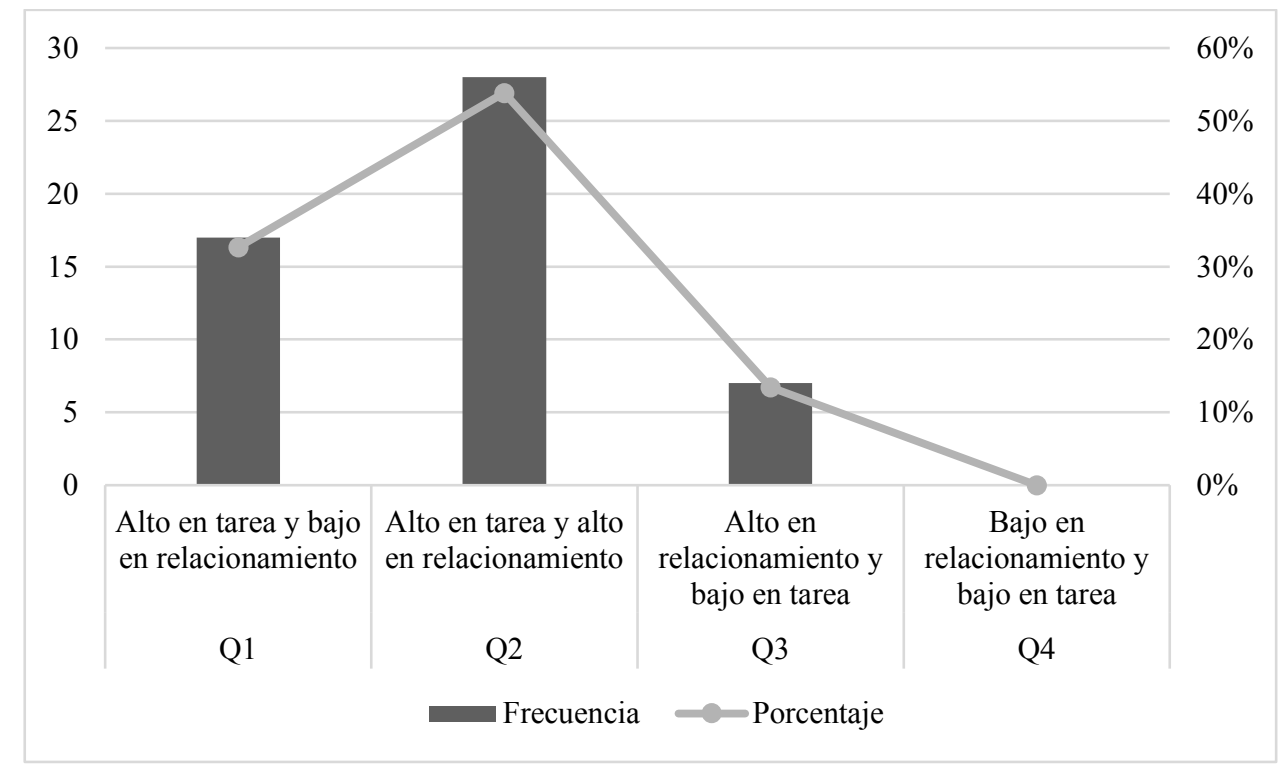

Figura 2: Frecuencia y porcentaje de resultados totales $Q$.

Fuente: (Cooperativa de Ahorro y Crédito San Francisco Ltda., 2020)

\subsection{Propuesta de Intervención}

La población mayoritaria según los resultados una vez realizado el estudio son 28 colaboradores están ubicados en Q2 (Aprendiz - Desilusionado) como se indica en la Figura 3 dan a notar que necesitan del estilo de liderazgo "dirección y de apoyo" para desenvolverse correctamente en sus puestos de trabajo y desarrollar adecuadamente sus funciones (Hernández \& Rodríguez, 2016).

Este estilo de liderazgo "dirección y de apoyo" se refleja debido que la población mayoritaria 28 colaboradores están en el rango de edad de 23 a 33 años de ambos sexos (Goleman, 2016).

Este grupo poblacional necesita un estilo de "Liderazgo Coach", el cual se caracteriza por ya ayuda que brinda el líder al colaborador o compañero de equipo de trabajo, uno de los aportes que este tipo de líder consigue es a encontrar las falencias - errores, para luego brindar un proceso de enseñanza y de esta manera se logré potencializar su talento (Pazmiño, Beltrán, \& Gallardo, 2016).

Además, se puede mencionar que mayoría de los colaboradores de la Cooperativa de Ahorro y Crédito El Sagrario Ltda. necesitan de un estilo de "Liderazgo Afiliativo" ya que con esta característica de afiliación empresarial el líder logra desarrollar empatía en los colaboradores mejorando así el clima y cultura organizacional, evitando que en el ambiente laboral se generen riesgos psicosociales para que este espacio laborar sea en donde se desarrolla el colaborador (Domínguez, 2019). 


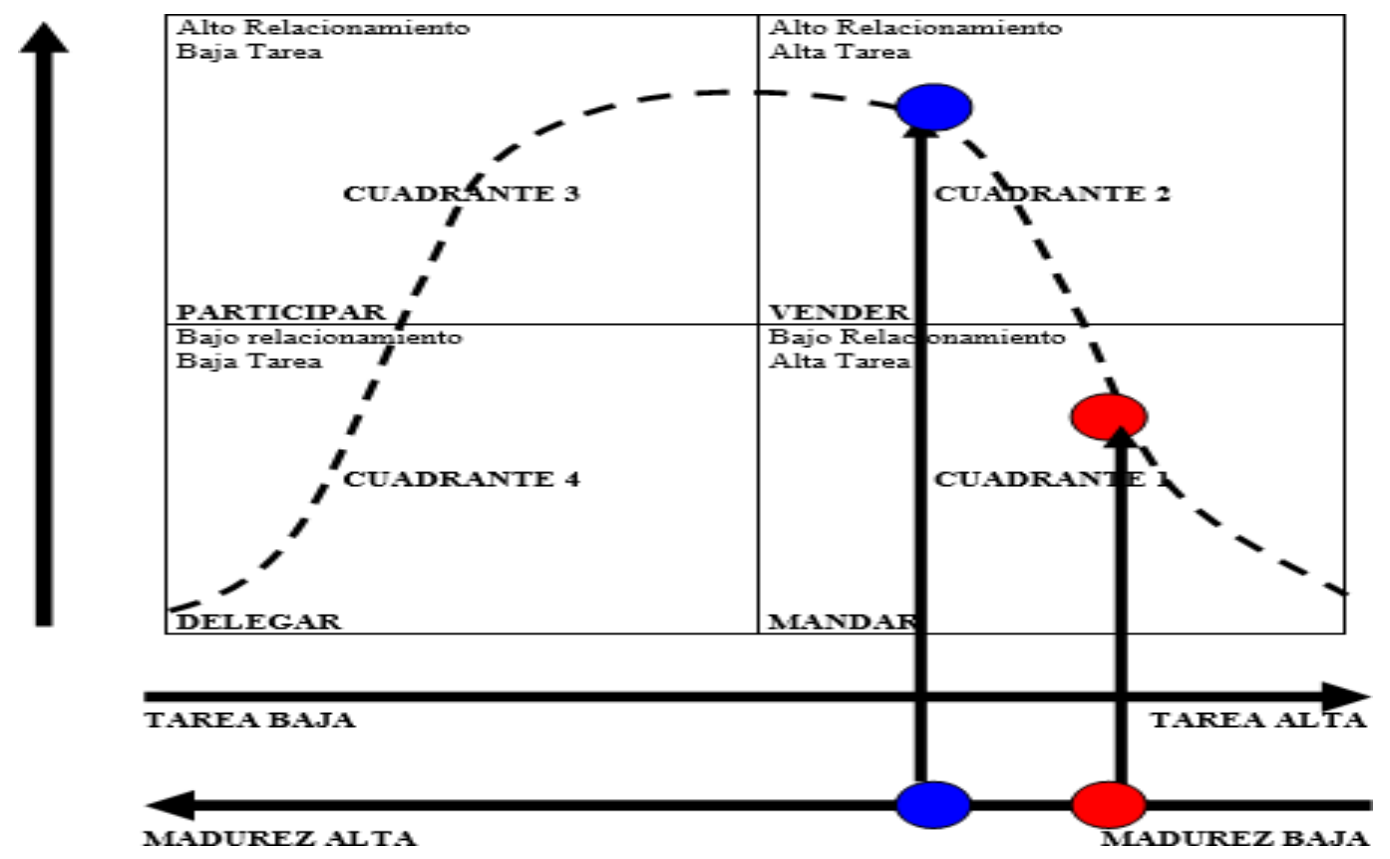

Figura 3. Estilo de liderazgo, según resultados de los colaboradores de la Cooperativa de Ahorro y Crédito El Sagrario Ltda.

Fuente: (Goleman, 2016)

Hay que mencionar de igual manera que el estilo de liderazgo que no se desarrolla en la Cooperativa El Sagrario Ltda. Se encuentra ubicado en el Q4 correspondiente al estilo de liderazgo "Triunfador - Independiente", lo que da a entender que los colaboradores de la institución no se sienten apoyo por parte de la organización, lo que conlleva a no desarrollar autonomía que se requiere para cumplir sus funciones en su puesto de trabajo, siempre deben están controlados por alguien.

Estos resultados arrojados de liderazgo en la empresa pese a que existe cierta tendencia de Q4 y Q2 en tiempos COVID-19, hace que se logre el 0\% de despido es decir que se mantenga el $100 \%$ de los colaboradores. A demás con estos estudios de liderazgo hace que a futuro se proponga planes de calidad de vida lo que en cuestión de manejo de RRHH y manejo de personas es beneficioso porque va causar un impacto positivo en otras personas que quieran pertenecer a la cooperativa. 


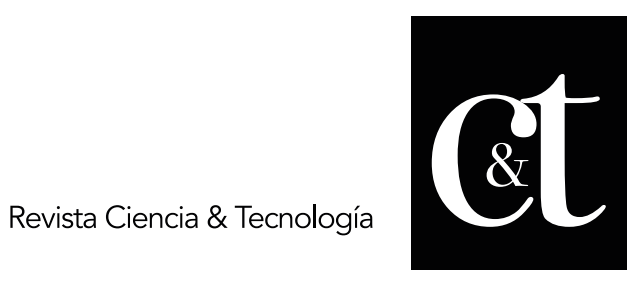

No. 32, 31 de octubre de 2021

ISSN impreso: 1390 - 6321

ISSN online: 2661 - 6734

Se ha logrado disminuir factores de riesgo psicosocial $5 \%$ de monotonía, $1 \%$ de nivel de estrés y $1 \%$ de burnout y $1 \%$ de burnout; y se logró incrementar un $16 \%$ de desempeño laborar con esta técnica de intervención hacia los colaboradores.

\section{Conclusiones y recomendaciones Conclusiones}

Realizada la evaluación de la prueba de Liderazgo Situacional de Hersey y Blanchard se ha obtenido: 28 colaboradores (54\%) se están desarrollando en un estilo de liderazgo poco apropiado para los colaboradores y para la institución; 17 colaboradores (33\%) desarrollan un estilo de liderazgo principiante necesitando la intervención y apoyo de la dirección; 7 colaboradores (13\%) necesitan más apoyo que dirección.

Con los resultados propuestos de liderazgo y debido a los tiempos de COVID 19 la Cooperativa EI Sagrario Ltda, se crearon nuevas políticas empresariales, se capacitó a los líderes para que se menore en el manejo del personal y los colaboradores de la institución tuvieron que adaptarse a las nuevas políticas que se desarrollaron dentro de la organización.

No se registraron despidos dentro de la institución debido a que todos los colaboradores se adaptaron a la situación que se atraviesa hoy en día, por tal motivo, Gerencia General decidió continuar con los 52 colaboradores que se encuentran trabajando en la institución se planteó nuevos horarios de trabajo, reestructuración de remuneraciones y otros.

\section{Recomendaciones}

El liderazgo empresarial más aun en tiempos de COVID-19 se debe evaluar cada año como lo indica el Ministerio de Trabajo del Ecuador con la finalidad de obtener resultados e ir tomando medidas preventivas y correctivas a tiempo, entre los colaboradores de la organización en caso de ser necesario.

El proceso de evaluación del liderazgo empresarial en tiempos de COIVD-19 debe considerar cambios internos y externos en caso de existirlos, con la finalidad de que los resultados no tengan tendencia positiva ni negativa es decir la información sea lo más real posible.

Esta metodología se recomienda aplicar en todas las organizaciones del ámbito administrativo, operativo o mixto debido a su factibilidad y adaptabilidad a cada una de las organizaciones.

Para finalizar es importante mencionar de acuerdo a los resultados la implementación de programas de liderazgo organizacional en donde se lleve a cabo un programa formativo enfocado al liderazgo con su respectiva planificación, seguimiento y control, basado en los principios y valores que debe tener un buen líder, enfocado en formación de equipos de trabajo. 


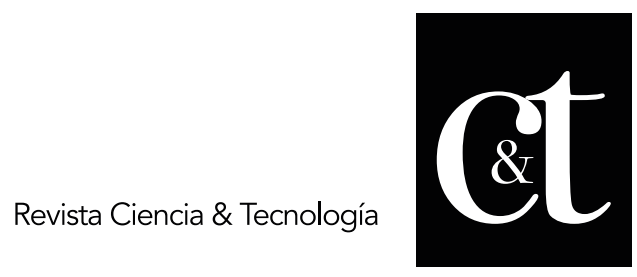

No. 32, 31 de octubre de 2021

ISSN impreso: 1390 - 6321

ISSN online: 2661 - 6734

\section{Referencias bibliográficas}

Ali Raza, S., \& Sikandar, A. (2018). Impact of Leadership Style of Teacher on the Performance of Students: An Application of Hersey and Blanchard Situational Model. Bulletin of Education and Research, 73-94.

Ariawan, R., \& Wahyuni, A. (2019). The effect of applying TPS type cooperative learning model assisted by SPSS software on students' skills in IT-based statistical data analysis course. Journal of Physics: Conference Series (pág. 8). Estados Unidos: IOP Publishing.

Cooperativa de Ahorro y Crédito San Francisco Ltda. (10 de 03 de 2020). Evaluación de Liderazgo Situacional de Hersey y Blanchard. Evaluación de Liderazgo Situacional de Hersey y Blanchard. Ambato, Tungurahua, Ecuador: Cooperativa de Ahorro y Crédito San Francisco Ltda.

Domínguez, M. (2019). El Liderazgo. Perú: Instituto IDEMA.

Freire, E. (2020). "LIDERAZGO ORGANIZACIONAL Y DESEMPEÑO LABORAL DE LOS COLABORADORES DE LA COOPERATIVA DE AHORRO Y CRÉDITO EL SAGRARIO $\angle T D A^{\prime \prime}$. Ambato: Universidad Técnica de Ambato.

García, J. (2015). LIDERAZGO ORGANIZACIONAL EN LOS SERVICIOS DE SALUD. Exégesis, 1-10.

Goleman, D. (2016). "El líder resonante crea más" - Estilos de Liderazgo. . Revista de estudios empresariales, 75-76.

Hernández, M., \& Rodríguez, Y. (2016). La Dirección Estratégica y el Cuadro de Mando Integral como herramienta de apoyo a la Gestión Empresarial en la Empresa de Proyectos de Arquitectura e Ingeniería de Matanzas. Revista de Arquitectura e Ingeniería, 1-9.

Hersey, P., \& Blanchard, K. (2005). Liderazgo Situacional. Metamanagers, 1-15.

IBM Corporation. (2012). Guia breve de IBM SPSS Statistics 21. Estados Unidos: GSA ADP Shedule Contract - IBM Corp.

Izquierdo, R., Novillo, L., \& Mocha, J. (2017). El liderazgo en las microempresas familiares, desafíos y metas. Universidad y Sociedad, 89-94.

Makovsky, P. (2014). Cómo llegar a ser un líder existoso. LIGS UNIVERSITY, 19.

Mantulak, M., \& Hernández, G. (2018). Capacidades que contribuyen al pensamiento estratégico. Un enfoque en la gestión tecnológica en PyMEs de la madera. Ingeniería Indutrial, 160-169.

Meliá, J. L., Nogareda, C., Lahera, M., Duro, A., Peiró, J. M., Salanova, M., \& Gracia, D. (2016). Principios Comunes para la Evaluación de los Riesgos Psicosociales en la Empresa. Perspectivas de intervención en riesgos psicosociales. Evaluación de riesgos, 13-36.

Mosquera, A., Castillo, R., \& Portilla, M. (2017). La responsabilidad social empresarial. Acciones institucionales en la gestión estratégica curricular. Entramado, 13.

Pazmiño, G., Beltrán, M., \& Gallardo, W. (2016). LOS ESTILOS DE LIDERAZGO Y SUINFLUENCIA EN EL DESARROLLO EMPRESARIAL: CASO PYMES DE LA PROVINCIA DE TUNGURAHUA - ECUADOR. Pontificia Universidad Católica del Ecuador, 16.

Pinto, Y., \& Gutiérrez, T. (2015). EFECTIVIDAD DE LOS ESTILOS DE LIDERAZGO SITUACIONAL DESDE UNA APROXIMACIÓN DEL MODELO DE HERSEY Y BLANCHARD. Revista Arjé, 11.

Rexhepi, A., Sogojeva, H., Rexhepi, B., \& Lekiqi, B. (2020). Leadershipstyles in Kosovo businesses. Technium, 12. 
Thompson, G., \& Glaso, L. (2018). Leadership \& Organization Development Journal. emeraldinsight, 20.

Turbay, M. (2013). Liderazgo e innovación organizacional. Psicología desde el Caribe, 5.

$\mathrm{Vu}, \mathrm{H}$. (2020). Empoderamiento de los empleados y liderazgo empoderador: una revisión de la literatura. Technium, 9.

Ward, J. (2012). El crecimiento de la empresa familiar: retos específicos y mejores prácticas. Recuperado de: https://cdn.ymaws.com/ffi.siteym.com/resource/resmgr/best_of_fbr_spanish/fbresp_ward_elcrecimiento.pd $f, \quad$ 9. Obtenido de https://cdn.ymaws.com/ffi.siteym.com/resource/resmgr/best_of_fbr_spanish/fbresp_ward_elcrecimiento.pd $\mathrm{f}$ 